\title{
Extraordinary Opportunity: Molecular Targets of Prevention and Treatment
}

National Cancer Institute

\section{Source}

National Cancer Institute. Extraordinary Opportunity: Molecular Targets of Prevention

and Treatment. NCl Thesaurus. Code C16138.

Goal: we now have very focused targets ag ainst which to direct prevention and therapy efforts. The Molecular Targets Initiative will seek to discover and develop novel prevention, therapeutic, diagnostic, and imaging agents based on our understanding of these targets. ( $\mathrm{NCl}$ Web) 